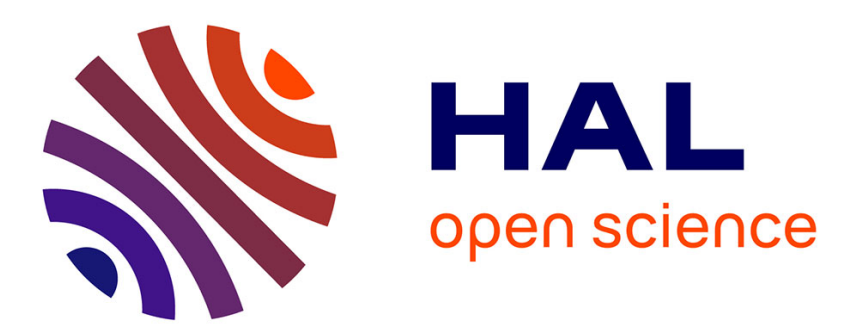

\title{
Human-Assisted Grasping for Manipulation of Biological Cells
}

\author{
Jiuyun Li, Quang Minh Ta, Chao Liu, Chien Chern Cheah
}

\section{To cite this version:}

Jiuyun Li, Quang Minh Ta, Chao Liu, Chien Chern Cheah. Human-Assisted Grasping for Manipulation of Biological Cells. IECON 2021 - 47th Annual Conference of the IEEE Industrial Electronics Society, Oct 2021, Toronto, Canada. pp.1-6, 10.1109/IECON48115.2021.9589144 • lirmm-03442315

\section{HAL Id: lirmm-03442315 https://hal-lirmm.ccsd.cnrs.fr/lirmm-03442315}

Submitted on 23 Nov 2021

HAL is a multi-disciplinary open access archive for the deposit and dissemination of scientific research documents, whether they are published or not. The documents may come from teaching and research institutions in France or abroad, or from public or private research centers.
L'archive ouverte pluridisciplinaire HAL, est destinée au dépôt et à la diffusion de documents scientifiques de niveau recherche, publiés ou non, émanant des établissements d'enseignement et de recherche français ou étrangers, des laboratoires publics ou privés. 


\title{
Human-Assisted Grasping for Manipulation of Biological Cells
}

\author{
Jiuyun Li, Quang Minh Ta, Chao Liu, and Chien Chern Cheah
}

\begin{abstract}
Optical tweezers have received significant attention in the past few decades and many techniques have been proposed to achieve assorted manipulation tasks on cells or micro-objects. While traditional techniques for optical tweezers utilize laser beams for direct trapping and manipulation of cells, several approaches have also been proposed to achieve grasping and manipulation of cells. Most grasping approaches, however, have failed to solve the existing difficulties in the grasping process of cells due to limited sensing capability and presence of Brownian perturbation in the micro world. In this paper, a novel human-assisted grasping technique for manipulation of biological cells is proposed to facilitate the grasping process of biological cells. In the proposed technique, several microbeads are first trapped and used as fingertips to perform a grasping task on a biological cell. Based on human observation, the positions of the laser beams that trap the microbeads are remotely and simultaneously controlled through a touchscreen, and thus generating a grasping formation of the trapped microbeads to grasp the cell. After the cell has been grasped, a simple region control technique is utilized for automated manipulation of the grasped cell. This paper offers a flexible, reliable and efficient approach for grasping and manipulation of biological cells, and thus extending the feasible applications of optical tweezers. The stability of the control system for manipulation of the grasped cell is investigated by using a Lyapunov approach, and the feasibility of the proposed human-assisted grasping and manipulation technique is demonstrated by experiment result.
\end{abstract}

\section{INTRODUCTION}

Optical tweezers play a vital role in molecular biology due to their capability to manipulate biological cells precisely and effectively. The pioneer work can be traced to [1]. In 1970, A. Ashkin discovered the ability of optical tweezers and demonstrated the experiment of utilizing single-beam force for trapping and levitating micro-particles in liquids and gas.

The operations of optical tweezers consist of trapping the target cell or micro-objects and moving the trapped cell or micro-objects. Earlier studies and applications have been focusing on the optical manipulation of single cell or microobject [2]- [4]. Later, a few techniques have been proposed for generation of multiple optical traps, such as holographic optical tweezers [5], scanning method [6], and acousto-optic deflectors [7]. Various techniques have been then developed for manipulation of multiple cells of micro-objects [8]- [11].

For many years, only direct trapping and manoeuvring of cells and micro-objects using laser beams had been

This work was supported by the Agency For Science, Technology and Research of Singapore (A*STAR), under the AME Individual Research Grants 2017 (Ref. A1883c0008).

Jiuyun Li, Quang Minh Ta, and Chien Chern Cheah are with the School of Electrical and Electronic Engineering, Nanyang Technological University, Singapore 639798. Chao Liu is with LIRMM, University of MontpellierCNRS, Montpellier, France. Email: E200041@e.ntu.edu.sg established. However, there exist many types of cells and micro-objects that cannot be directly trapped using the laser beams. Besides, direct trapping for manipulation of cells using laser beams may cause photo-damages to the trapped cells. To overcome this issue, several control techniques [12][14] and planning approaches [15], [16] have been proposed for indirect manipulation of target cells or micro-objects by using several trapped microbeads. In these methodologies [12]- [16], several microbeads were trapped by the laser beams and manually brought around the target cells or micro-objects. Grasping formations or grippers formed by the trapped microbeads were then generated to hold and move the cells or micro-objects. As manual grasping of target micro-objects by separately adjusting each trapped microbead sequentially is ineffective and time consuming, automated grasping approaches were then proposed in [17], [18]. In these approaches [17], [18], the grasping formations for the target cells or micro-objects were automatically generated by controlling the formation of the trapped microbeads.

In general, the automated grasping process of biological cells or objects in the micro-world is difficult due to the lack of feasible sensors at the micro-scale. In addition, this process is also challenged by the random Brownian motion of cells or micro-objects in the fluid media. In contrast, human, with visual ability, is able to perform the grasping tasks effectively by coordinating multiple fingers. In this paper, a novel human-assisted grasping technique for manipulation of biological cells is proposed to achieve grasping tasks by using several trapped microbeads. In the proposed technique, several microbeads are first trapped and used as fingertips to perform a grasping task on a biological cell. Based on human observation, the positions of the trapped microbeads are controlled by the user through a touchscreen to formulate a grasping formation around the cell to grasp it. After the cell has been grasped, a simple region control technique is utilized for automated manipulation of the grasped cell. In the grasping strategy, a touchscreen Graphical User Interface (GUI) is designed to realize the interaction between the positions of human's fingertips on the touchscreen and the positions of the laser beams in real-time. Data transfer and interactive operations are thus carried out between the user and the optical tweezers system through a Transmission Control Protocol (TCP). When the user changes the fingertips' positions on the touchscreen, the corresponding laser beams are driven accordingly so as to bring the fingertips for grasping of the target cell. Once the cell has been grasped, it is automatically driven to a desired region by using the fingertips. This study offers an effective way to grasp bio- 
logical cells by using multiple trapped microbeads. With the assistance of human, in terms of decision making and instant reaction, this paper offers a more reliable grasping approach which helps save time and reduce cell loss. The stability of the manipulation system is investigated by using a Lyapunov stability approach, and the feasibility of the proposed humanassisted grasping technique for manipulation of biological cells is demonstrated by experiment.

\section{PROBLEM Formulation}

Optical tweezers have been proven useful in many micromanipulation tasks because of their high precision and capability in manipulation of multiple cells or micro-objects simultaneously. A typical setup of an optical tweezers system is shown in Fig. 1.

This paper presents a novel human-assisted system for grasping and manipulation of biological cells utilizing multiple trapped microbeads. In general, the grasping process of biological cells or objects in the micro-world is difficult due to the lack of feasible sensors at the micro-scale. In addition, this process is also challenged by the random Brownian motion of cells or micro-objects in the media. Human, in terms of visual capability, is able to perform the grasping task effectively. Based on human observation, the positions of the laser beams that trap the microbeads are remotely and simultaneously controlled through a touchscreen, and thus generating a grasping formation of the trapped microbeads to grasp the cell. To achieve this objective, a human-robot interface for interaction between the touchscreen and the optical tweezers system is designed, and a communication protocol is created for data transmission between them. The movements of human's fingertips on the touchscreen are thus transferred to the optical tweezers system in order to move the corresponding trapped microbeads for completion of the grasping task. An illustration of the grasping process using

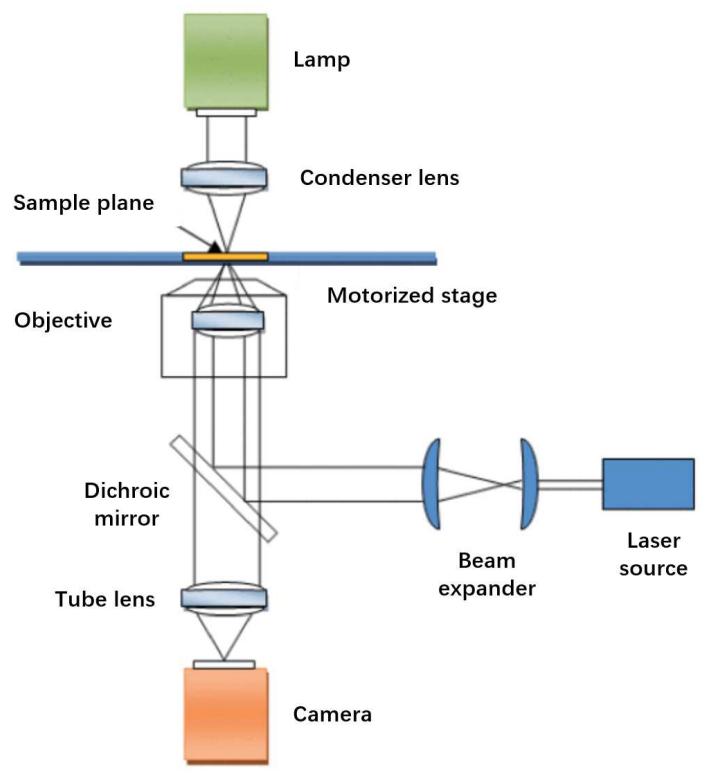

Fig. 1. An illustration of an optical tweezers system

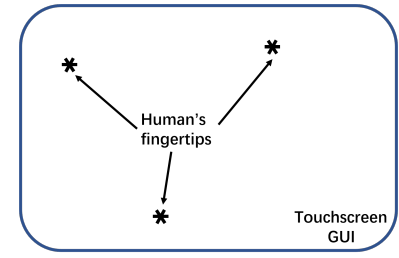

(a) The positions of human's fingertips in the work space of the touchscreen are corresponding to the positions of laser beams in the working space for grasping and manipulation of the cell.

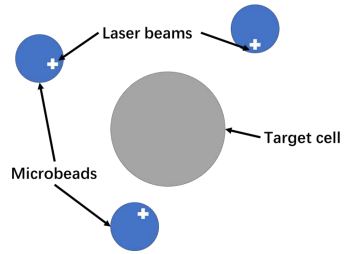

(b) Initial positions of microbeads and the target cell.

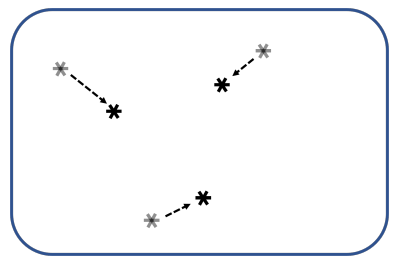

(c) The movements of the fingertips on the touchscreen leads to the movements of the corresponding laser beams.

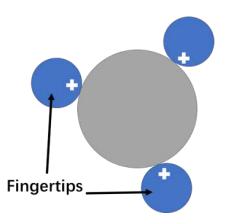

(d) The grasping formation of the trapped microbeads generated by user to grasp the target cell.
Fig. 2. Illustration of the proposed grasping process with the aid of the designed GUI. Based on human observation, user is able to control the positions of the trapped microbeads by adjusting the fingertips' positions on the touchscreen, which is connected to the optical tweezers system through a TCP connection. The laser beams are initially assigned and then moved to the nearest positions of human's fingertips. When user changes the fingertips' positions on the touchscreen, the corresponding laser beams are driven accordingly so as to position the fingertips for grasping of the target cell.

several trapped microbeads is shown in Fig. 2. After the cell has been grasped, a simple region control technique is utilized for automated manipulation of the grasped cell.

In the proposed approach, once the target cell has been grasped by using the fingertips, it is automatically transported towards a desired region by using the fingertips. The dynamics of the trapped microbeads in the manipulation process are described as [17]:

$$
-\boldsymbol{D}_{f} \dot{\boldsymbol{p}}_{i}+\boldsymbol{S}\left(\boldsymbol{q}_{i}-\boldsymbol{p}_{i}\right)-\boldsymbol{f}_{i}=0,
$$

where $i=1, \ldots, n$ with $n$ represents the number of microbeads to grasp and manipulate the target cell. The vectors $\boldsymbol{q}_{i} \in \mathcal{R}^{2}$ indicate the positions of the laser beams while $\boldsymbol{p}_{i} \in \mathcal{R}^{2}$ indicate the positions of the microbeads. The matrices $\boldsymbol{D}_{f} \in \mathcal{R}^{2 \times 2}$ and $\boldsymbol{S} \in \mathcal{R}^{2 \times 2}$ denote the damping matrix and the trapping stiffness respectively, which are diagonal and positive definite matrices. In (1), $\boldsymbol{f}_{i} \in \mathcal{R}^{2}$ is the contact force exerted on the target cell by the $i^{t h}$ microbead. In micromanipulation, the inertia terms can be ignored due to the low Reynolds number.

The dynamics of the target cell in the grasping formation is specified as:

$$
-\boldsymbol{D}_{o} \dot{\boldsymbol{y}}+\sum_{i=1}^{n} \boldsymbol{f}_{i}=0
$$

where $y \in \mathcal{R}^{2}$ represents the position of the target cell. All the positions of the microbeads and the target cell are 

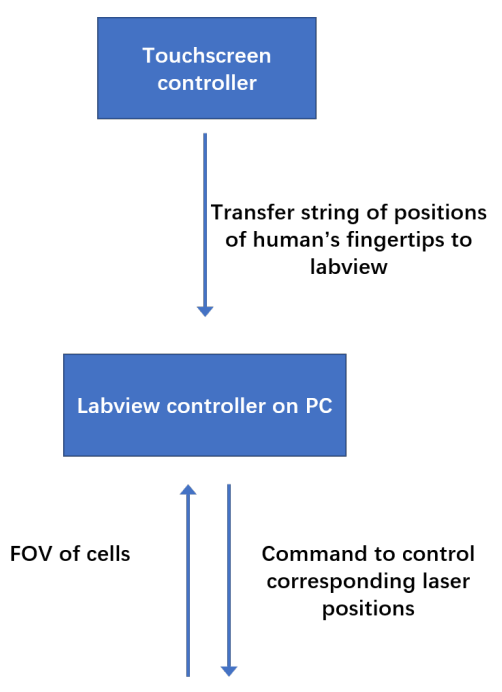

Optical tweezers

Fig. 3. Block diagram illustrating the interaction between the three devices for manipulation of the laser beams in the grasping process.

specified with respect to a reference frame attached to the field of view of the camera. The matrix $\boldsymbol{D}_{o} \in \mathcal{R}^{2 \times 2}$ denotes a diagonal and positive definite damping matrix. Every single microbead $i$ exerts a contact force $\boldsymbol{f}_{i}$ on the manipulated cell, and the summation of the contact force from all the microbeads on the target cell is thus described as $\sum_{i=1}^{n} \boldsymbol{f}_{i}$.

\section{HUMAN-ASSISTED GRASPING FOR GRASPING OF BIOLOGICAL CELLS}

This research proposes a technique to grasp the target cell through human-computer interaction. Based on human observation, user is able to control the positions of the trapped microbeads by adjusting the fingertips' positions on the touchscreen, which is connected to the optical tweezers system through a TCP connection. The communication system consists of a touchscreen controller, optical tweezers and LABVIEW control unit. In the proposed technique, the touchscreen controller is coordinated correspondingly to the working space for grasping of cells using trapped microbeads. Each laser beam is initially assigned and then moved to the nearest corresponding position of the fingertips on the touchscreen. When user changes the fingertips' positions on the touchscreen, the corresponding laser beams are driven accordingly so as to bring the fingertips for grasping of the target cell. The data of the fingertips' positions were delivered to the LABVIEW program through a TCP connection. Finally, transition commands were sent to the laser emitter of the optical tweezers system so as to drive the laser beams accordingly. Fig. 3. provides the intercorrelations among the three devices for human-assisted grasping of biological cells.

An illustration for the process of connection establishment between the touchscreen and the LABVIEW control unit is shown in Fig. 4, where Transmission Control Block
(TCB) is utilized to encapsulate data for different devices to communicate. Aimed at the communication across devices, TCP is established in the transport layer protocol to ensure the stability and accuracy of the transmission. TCP is a connection-oriented, reliable transport-level communication protocol based on byte stream, with flow control and error control. In this approach, the touchscreen controller is specified as the server and LABVIEW control unit is designated as the client. On completion of three handshakes, a TCP connection is established between the server and the client. First, a request for telecommunication is sent from the client to the server. In order to check the feasibility of the connection, a confirmation of the receipt for the request message is dispatched from the server. Finally, the confirmation is received by the client. After the connection is established, the real-time position data of the laser beams from the server in the form of a string is sent to the control device of the optical tweezers system. Disconnection process is broken after four waves, which is similar to the establishment procedure of connection. Therefore, the reliability of the transmission process is proved and meanwhile the rate of packet loss is ensured to satisfy the manipulation requirements of lasers in real-time.

By application of a TCP connection, the stability of the communication system is achieved, and performance is prompted on account of fast retransmission and delayed reply at the same time.

\section{MAnipulation of THE GRASPED OBJECT With A REGION CONTROL TECHNIQUE}

In the previous section, a description of the communication model for grasping of the target cell was presented. This section demonstrates the use of a region control technique for transportation of the grasped cell.

A desired region for manipulation of the cell can be

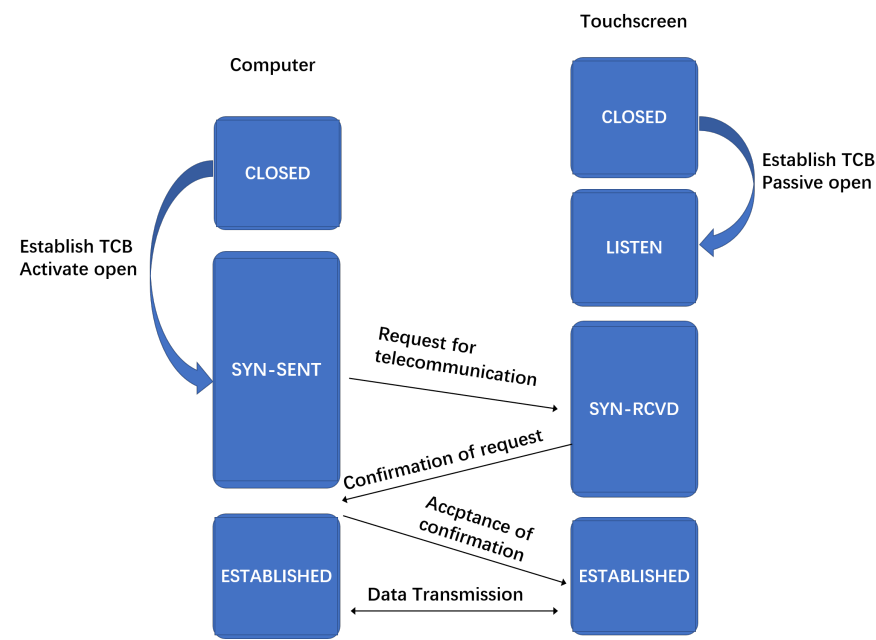

Fig. 4. Process of TCP establishment between the touchscreen and the LABVIEW control unit 
specified as [14]:

$$
f(\boldsymbol{y})=\frac{\left(y_{1}-y_{o 1}\right)^{k}}{r_{1}^{k}}+\frac{\left(y_{2}-y_{o 2}\right)^{k}}{r_{2}^{k}}-1 \leq 0,
$$

where $\boldsymbol{y}_{o}=\left(y_{o 1}, y_{o 2}\right)^{T}$ is the central point of the specified region, and $r_{1}, r_{2}$ are the semi-major axis and semi-minor axis of the superellipse respectively, and $k$ is the order of the superellipse. When $r_{1}=r_{2}=R$ and $k=2$, the desired region becomes a circle with a radius $R$.

Then we can derive an energy function associated with the desired region as:

$$
E=\frac{\beta}{\alpha} \max [0, f(\boldsymbol{y})]^{\alpha},
$$

where $\alpha$ and $\beta$ are positive constants. The parameter $\alpha$ must be an even number and larger than 2 , so that the energy function $E$ is twice-differentiable.

By differentiating the energy function $E$ in (4), the control error of the target cell with respect to the desired region can be obtained as:

$$
\begin{aligned}
\Delta \boldsymbol{\delta} & =\frac{\partial E}{\partial(\Delta \boldsymbol{y})} \\
& =\beta \max [0, f(\boldsymbol{y})]^{\alpha-1}\left(\frac{\partial f(\boldsymbol{y})}{\partial(\Delta \boldsymbol{y})}\right),
\end{aligned}
$$

with $\Delta \boldsymbol{y}=\boldsymbol{y}-\boldsymbol{y}_{o}$.

When the manipulated cell is outside the desired region, the error $\Delta \delta$ in (5) drives the cell towards the desired region. Conversely, when the cell is inside the region, $\Delta \boldsymbol{\delta}=0$, and thus the cell remains in the region.

From (1), the dynamics of $n$ microbeads in the grasping formation is described as:

$$
-\boldsymbol{D} \dot{\boldsymbol{p}}+\boldsymbol{K}(\boldsymbol{q}-\boldsymbol{p})-\boldsymbol{f}=0,
$$

with $\boldsymbol{D}=\operatorname{diag}\left\{\boldsymbol{D}_{f}, \ldots, \boldsymbol{D}_{f}\right\} \in \mathcal{R}^{2 n \times 2 n}$, and $\boldsymbol{K}=$ $\operatorname{diag}\{\boldsymbol{S}, \ldots, \boldsymbol{S}\} \in \mathcal{R}^{2 n \times 2 n}$ are diagonal matrices, and $\boldsymbol{p}=$ $\left[\boldsymbol{p}_{1}^{T}, \boldsymbol{p}_{2}^{T}, \ldots, \boldsymbol{p}_{n}^{T}\right]^{T} \in \mathcal{R}^{2 n}$, and $\boldsymbol{f}=\left[\boldsymbol{f}_{1}^{T}, \boldsymbol{f}_{2}^{T}, \ldots, \boldsymbol{f}_{n}^{T}\right]^{T} \in$ $\mathcal{R}^{2 n}$.

Multiplying (6) with a matrix $\boldsymbol{I}_{n}=\left[\boldsymbol{I}_{2}, \ldots, \boldsymbol{I}_{2}\right] \in \mathcal{R}^{2 \times 2 n}$ in which $\boldsymbol{I}_{2} \in \mathcal{R}^{2 \times 2}$ is an identity matrix, we obtain:

$$
-\boldsymbol{I}_{n} \boldsymbol{D} \dot{\boldsymbol{p}}+\boldsymbol{I}_{n} \boldsymbol{K}(\boldsymbol{q}-\boldsymbol{p})-\boldsymbol{I}_{n} \boldsymbol{f}=0 .
$$

Note that:

$$
\boldsymbol{I}_{n} \boldsymbol{f}=\sum_{i=1}^{n} \boldsymbol{f}_{i} .
$$

Replacing (2) into (7) using (8) and rewriting to get:

$$
\boldsymbol{I}_{n} \boldsymbol{D} \dot{\boldsymbol{p}}+\boldsymbol{D}_{o} \dot{\boldsymbol{y}}+\boldsymbol{I}_{n} \boldsymbol{K} \boldsymbol{p}=\boldsymbol{I}_{n} \boldsymbol{K} \boldsymbol{q} .
$$

Assuming that the center of $n$ microbeads matches up with the center of the target cell, the velocity of the cell is thus derived as:

$$
\dot{\boldsymbol{y}}=\frac{1}{n} \sum \dot{\boldsymbol{p}}_{i}=\frac{1}{n} \boldsymbol{I}_{n} \dot{\boldsymbol{p}} .
$$

It is noted that $\boldsymbol{I}_{n} \boldsymbol{D}=\boldsymbol{D}_{f} \boldsymbol{I}_{n}$, and $\boldsymbol{I}_{n} \boldsymbol{K}=\boldsymbol{S} \boldsymbol{I}_{n}$. The dynamic equation between the target cell and the trapped microbeads in (9) can be rewritten as:

$$
\left(n \boldsymbol{D}_{f}+\boldsymbol{D}_{o}\right) \dot{\boldsymbol{y}}+\boldsymbol{S} \boldsymbol{I}_{\boldsymbol{n}} \boldsymbol{p}=\boldsymbol{S} \boldsymbol{I}_{\boldsymbol{n}} \boldsymbol{q} .
$$

The controller for the laser-actuated system is now designed as:

$$
\boldsymbol{q}_{i}=\boldsymbol{p}_{i}-\frac{1}{n} \boldsymbol{G} \Delta \boldsymbol{\delta}
$$

where $\Delta \boldsymbol{\delta}$ is defined in (5), and $G \in \mathcal{R}^{2 \times 2}$ is a gain matrix, which is diagonal and positive definite.

Substituting (12) into (11), the overall closed-loop equation is described as:

$$
\left(n \boldsymbol{D}_{f}+\boldsymbol{D}_{o}\right) \dot{\boldsymbol{y}}+\boldsymbol{S} \boldsymbol{G} \boldsymbol{\Delta} \boldsymbol{\delta}=0 .
$$

Then we can state the following theorem:

Theorem. The proposed controller given by (12) for automatic transportation of the grasped cell towards a desired region guarantees the stability of the closed-loop manipulation system. That is, $\Delta \boldsymbol{\delta} \rightarrow 0$ as $t \rightarrow \infty$, and the target cell is driven towards the desired region.

Proof. A Lyapunov-like candidate function to investigate the stability of the controlled system (13) is proposed as:

$$
V=E \geq 0,
$$

with $E$ is specified in (4).

Taking the derivative of $V$ in (14) with respect to time to obtain:

$$
\begin{aligned}
\dot{V} & =\dot{E} \\
& =\Delta \boldsymbol{\delta}^{T} \dot{\boldsymbol{y}} .
\end{aligned}
$$

From (13), it can be derived that:

$$
\dot{\boldsymbol{y}}=-\left(n \boldsymbol{D}_{f}+\boldsymbol{D}_{o}\right)^{-1} \boldsymbol{S} \boldsymbol{G} \boldsymbol{\Delta} \boldsymbol{\delta} .
$$

Replacing (16) into (15) to get:

$$
\dot{V}=-\boldsymbol{\Delta} \boldsymbol{\delta}^{T}\left(n \boldsymbol{D}_{f}+\boldsymbol{D}_{o}\right)^{-1} \boldsymbol{S} \boldsymbol{G} \boldsymbol{\Delta} \boldsymbol{\delta} .
$$

Since $\left(n \boldsymbol{D}_{f}+\boldsymbol{D}_{o}\right)^{-1} \boldsymbol{S} \boldsymbol{G}$ is diagonal and positive definite, thus $\dot{V} \leq 0$. As $\dot{V}=0$ implies that $\Delta \boldsymbol{\delta}=0$ as $t \rightarrow \infty$, therefore, according to LaSalle's Invariance Principle, the theorem is proved.

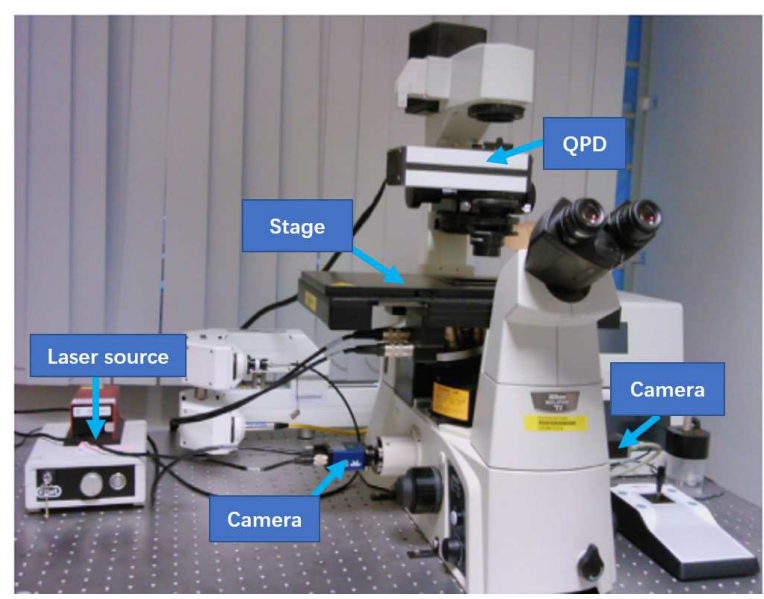

Fig. 5. An optical tweezers system consists of a microscope, a laser source, a motorized stage, and two cameras. 


\section{EXPERIMENT}

The proposed approach for human-assisted grasping and transportation of biological cells was implemented using the optical tweezers shown in Fig. 5. A Surface Pro 3 was utilized as the touchscreen controller for issuing transition instructions to the optical tweezers system. To achieve the communication for human-assisted grasping, a TCP connection between touch trace and optical tweezers was established on a Local Area Network (LAN), using custom software written in Python. During the grasping process, the positions of the laser beams were controlled by adjusting the positions of human's fingertips on the touchscreen. The trapped microbeads were thus driven accordingly to generate a grasping formation for the target cell. In the manipulation process, the proposed controller in (12) for transportation of the grasped cell was programmed in LABVIEW so as to drive towards a desired region.

In the following experiment, a cornflour particle with the diameter of approximately $10 \mu \mathrm{m}$ was employed as the target cell to be grasped and manoeuvred. Three polystyrene microbeads, with the diameters of $5 \mu \mathrm{m}$ and refractive indexes of 1.6, were selected as the fingertips to grasp the target cell after they had been trapped by the laser beams. Ultrapure water was chosen as the fluid medium for the experiment.

In the experiment, prior to the course of the grasping, three laser beams were generated by the optical tweezers system and each beam was allocated to trap one microbead. After that, a micro-hand consisting of three trapped microbeads was remotely controlled by human through a touchscreen, and thus forming a grasping formation to grasp the target cell. Data transmission in real-time was ensured by a TCP connection, thus the positions of human's fingertips on the touchscreen, with a designed GUI, can be transferred to the laser emitter. Preliminary work on TCP was undertaken by a TCP/IP socket, which bound the port and the IPv4 address from its network connection to supply connectivity among GUI and the optical tweezers system. A string of 18 bytes was sent to the port for every single touch, including 6

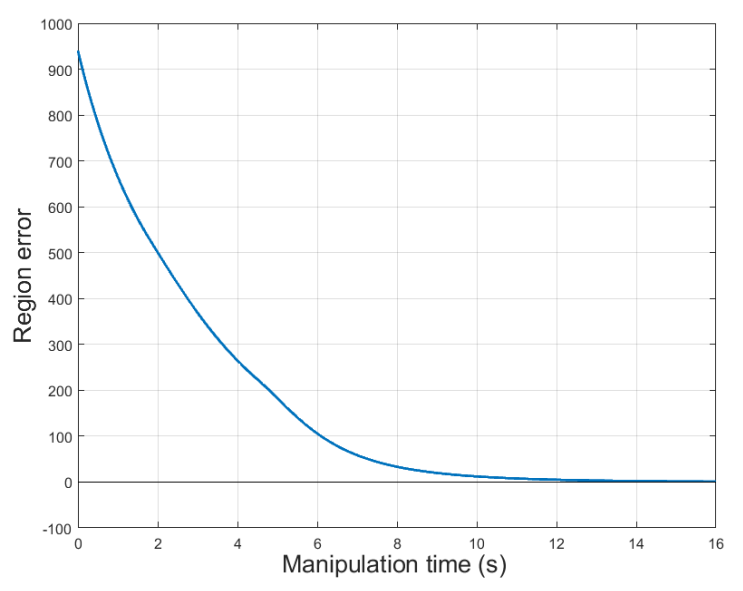

Fig. 6. Region error of the cornflour particle. coordinates from 3 fingertips. Once the grasping process had been completed, the cell was driven towards a circular area specified in (3). The power of each laser beam was set as $0.1 \mathrm{~W}$, and the control parameters for manipulation of the grasped corn-flour particle were selected as $\alpha=4, \beta=1$ to satisfy (4), and a positive definite gain matrix $\boldsymbol{G}$ in (12) was set to be $\boldsymbol{G}=\operatorname{diag}\left\{0.25 \times 10^{-3}, 0.25 \times 10^{-3}\right\}$. The region error of the grasped cornflour particle with respect to the desired region, which is specified as $\sqrt{\left(\Delta \boldsymbol{\delta}_{x}\right)^{2}+\left(\Delta \boldsymbol{\delta}_{y}\right)^{2}}$ with $\Delta \boldsymbol{\delta}=\left(\Delta \boldsymbol{\delta}_{x}, \Delta \boldsymbol{\delta}_{y}\right)^{T}$, is presented in Fig. 6. Different time instants of the grasping and transportation process were captured in Fig. 7.

\section{CONCLUSION}

This paper has proposed for the first time, a humanassisted grasping technique for manipulation of biological cells. In the proposed technique, several microbeads are first optically trapped by using laser beams. The trapped microbeads are then remotely and simultaneously controlled by human through a touchscreen in order to achieve the grasping task effectively. After the cell has been grasped, a simple region control technique is utilized for automated manipulation of the grasped cell towards a desired region. With the assistance of human, in terms of decision making and instant reaction, the proposed technique has offered a flexible, reliable and efficient methodology for grasping and manipulation of biological cells. The proposed technique in this paper has also contributed to the completion of optical tweezing and has significantly extended the feasible implementation of optical cell manipulation in various biological applications. The stability of the control system for manipulation of the grasped cell has investigated using a Lyapunov approach, and the feasibility of the the proposed human-assisted grasping and manipulation technique has been demonstrated by experiment result.

\section{REFERENCES}

[1] A. Ashkin. Acceleration and trapping of particles by radiation pressure. Physical Review Letters, vol. 24, pp. 156-159, 1970.

[2] A. Ashkin, and J. M. Dziedzic. Optical trapping and manipulation of viruses and bacteria. Science, vol. 235, pp. 1517-1520, 1987.

[3] S. C. Grover, A. G. Skirtach, R. C. Gauthier, and C. P. Grover. Automated single-cell sorting system based on optical trapping. Journal of biomedical optics, vol. 6, no. 1, pp. 14-22, 2001.

[4] X. Li and C. C. Cheah. Dynamic region control for robot-assisted cell manipulation using optical tweezers. IEEE International Conference on Robotics and Automation, pp. 1057-1062, 2012.

[5] E. Dufresne, G. Spalding, M. Dearing, S. Sheets, and D. Grier. Computergenerated holographic optical tweezers arrays. The Review of Scientific Instruments, vol. 72, pp. 1810-1816, 2001.

[6] K. Visscher, G. J. Brakenhoff, and J. J. Kroll. Micromanipulation by multiple optical traps created by a single fast scanning trap integrated with the bilateral confocal scanning laser microscope. Cytometry, vol. 14, pp. 105-114, 1993.

[7] K. C. Vermeulena, J. V. Mamerena, G. J. M. Stienen, E. J. G. Peterman, G. J. L. Wuite, and C. F. Schmidt. Calibrating bead displacements in optical tweezers using acousto-optic deflectors. The Review of Scientific Instruments, vol. 77, 2006.

[8] Q. Zhou, V. Sariola, K. Latifi, and V. Liimatainen. Controlling the motion of multiple objects on a chladni plate. Nature Communications, vol. 7, 2016. 


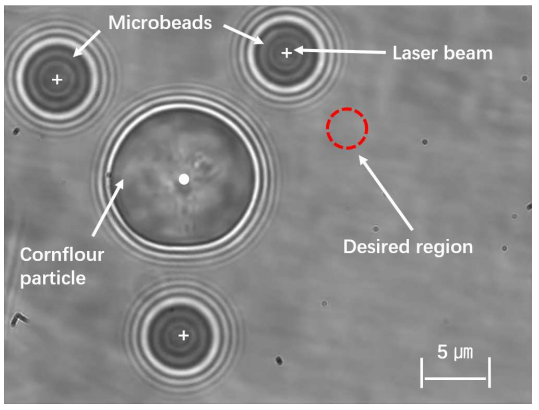

(a) $\mathrm{t}=0 \mathrm{~s}$. Initial positions.

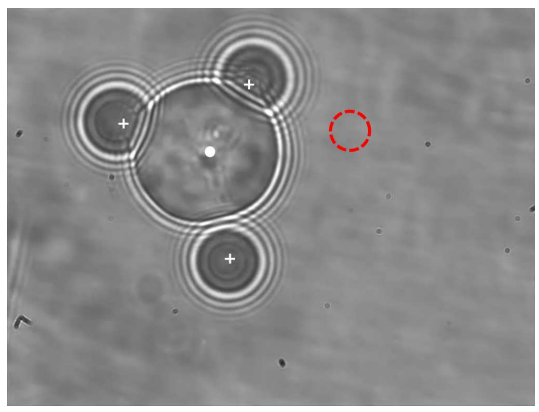

(d) $\mathrm{t}=5 \mathrm{~s}$.

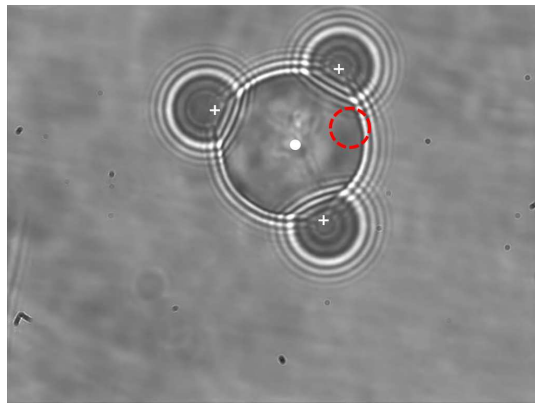

(g) $\mathrm{t}=13 \mathrm{~s}$

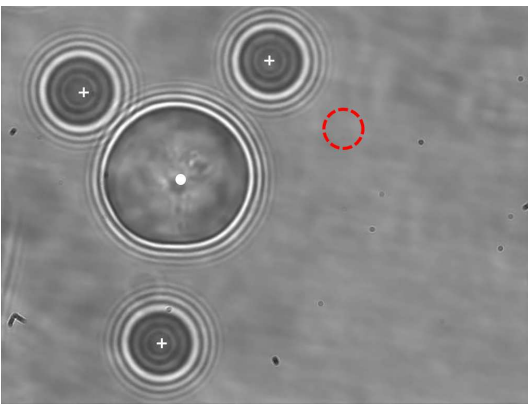

(b) $\mathrm{t}=1 \mathrm{~s}$. Based on human observation, the positions of the microbeads are controlled by adjusting the fingertips' positions on the touchscreen.

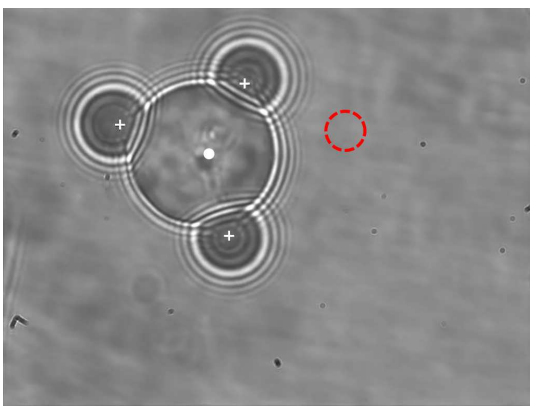

(e) $\mathrm{t}=6 \mathrm{~s}$. Completion of the grasping process.

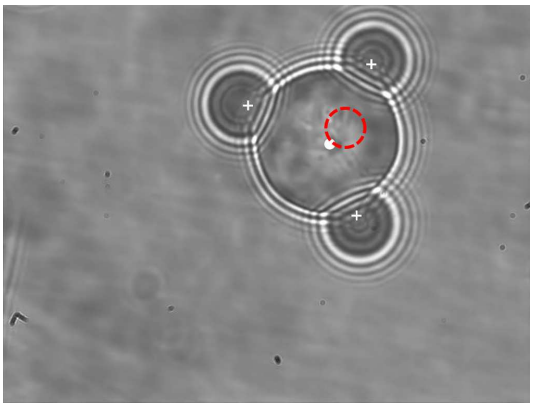

(h) $\mathrm{t}=17 \mathrm{~s}$.

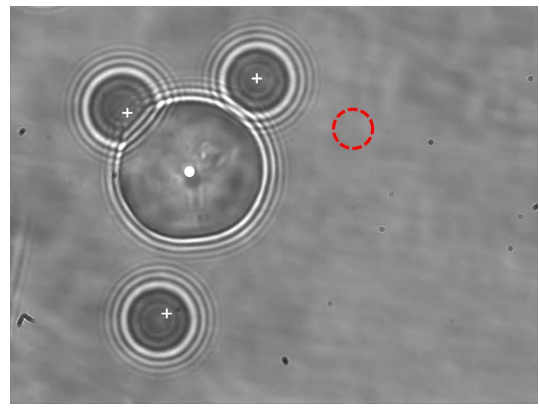

(c) $\mathrm{t}=2 \mathrm{~s}$. Due to Brownian perturbation, the target particle is randomly moving, which may cause difficulties for most existing techniques.

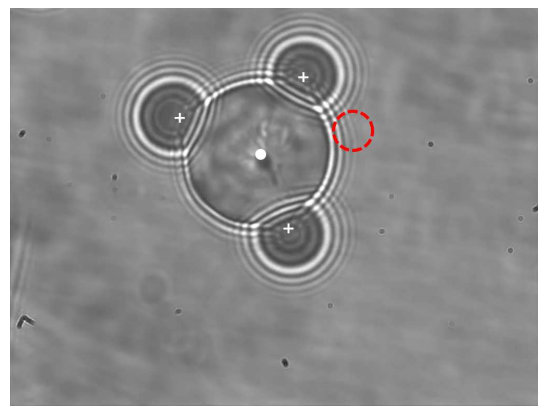

(f) $\mathrm{t}=9 \mathrm{~s}$. Manipulation of the grasped particle towards a desired region.

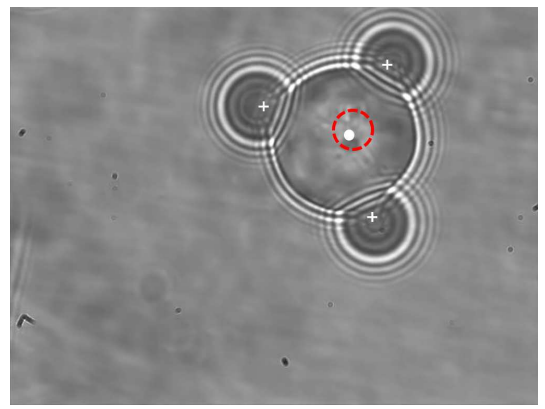

(i) $\mathrm{t}=20 \mathrm{~s}$. The grasped cornflour particle is driven towards the desired region.

Fig. 7. Experimental result for grasping and manipulation of a cornflour particle using the trapped microbeads. Three microbeads were first trapped and used as fingertips for grasping of the target particle. A grasping formation was generated by controlling the positions of fingertips through the touchscreen (Fig. 7(a)-7(e)). Fig. 7(f)-7(i) show the transportation process of the target particle towards a desired region.

[9] H. Chen and Y. Lou. Flocking multiple micro-particles with automatically controlled optical tweezers: Solutions and experiments. IEEE Transactions on Bio-medical Engineering, vol. 60, no. 6, pp. 15181527, 2013.

[10] F. Arai, K. Yoshikawa, T. Sakami, and T. Fukuda. Synchronized laser micro-manipulation of multiple targets along each trajectory by single laser. Applied Physics Letters, vol. 85, pp. 4301-4304, 2004.

[11] Q. M. Ta and C. C. Cheah. Stochastic control for optical manipulation of multiple microscopic objects. Automatica, vol. 89, pp. 52-64, 2018.

[12] Q. M. Ta and C. C. Cheah. Cooperative and mobile manipulation of multiple microscopic objects based on micro-hands and laser-stage control. Automatica, vol. 98, pp. 201-214, 2018.

[13] Q. M. Ta and C. C. Cheah. Stochastic Control for Orientation and Transportation of Microscopic Objects Using Multiple Optically Driven Robotic Fingertips. IEEE Transactions on Robotics, vol. 35, no. 4, pp. 861-872, 2019.

[14] Q. M. Ta and C. C. Cheah. Multi-agent Control for Stochastic Optical Manipulation Systems. IEEE/ASME Transactions on Mechatronics, vol. 25, no. 4, pp. 1971-1979, 2020.
[15] S. Chowdhury, A. Thakur, C. Wang, P. Svec, W. Losert, and S. K. Gupta. Automated manipulation of biological cells using gripper formations controlled by optical tweezers. IEEE Transactions on Automation Science and Engineering, vol. 11, no. 2, pp. 338-347, 2014.

[16] A. Thakur, S. Chowdhury, P. Svec, C. Wang, W. Losert, and S. K. Gupta. Indirect pushing based automated micromanipulation of biological cells using optical tweezers. International Journal of Robotics Research, vol. 33, no. 8, pp. 1098-1111, 2014.

[17] C. C. Cheah, Q. M. Ta, and R. Haghighi. Grasping and manipulation of a micro-particle using multiple optical traps. Automatica, vol. 68, pp. 216-227, 2016.

[18] Q. M. Ta and C. C. Cheah. Robotic Handling of Micro-Objects Using Stochastic Optically-Actuated End-Effector. IEEE Robotics and Automation Letters, vol. 6, no. 2, pp. 2930-2937, 2021. 